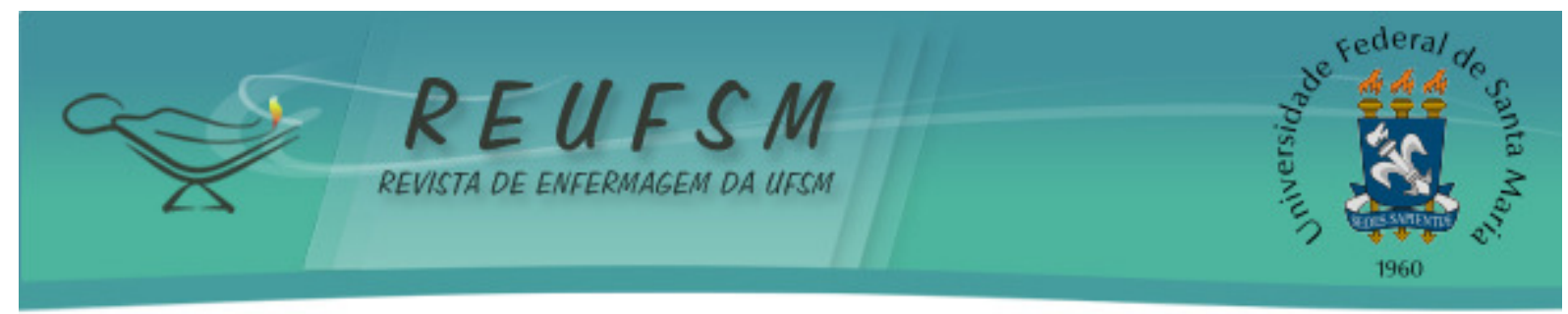

ARTIGO ORIGINAL

\title{
DIAGNÓSTICOS DE ENFERMAGEM APLICÁVEIS A PESSOAS COM PARAPLEGIA EM FASE INICIAL DE REABILITAÇÃO DOMICILIAR
}

\section{NURSING DIAGNOSIS APPLICABLE TO PEOPLE WITH PARAPLEGIA IN INITIAL PHASE OF HOME REHABILITATION}

\section{DIAGNÓSTICOS DE ENFERMERÍA PARA LAS PERSONAS CON PARAPLEJIA EN FASE INICIAL DE REHABILITACIÓN DOMICILIARIA}

Karenine Maria Holanda Cavalcante ${ }^{1}$

Zuila Maria de Figueirêdo Carvalho ${ }^{2}$ Francisco Moacir Pinheiro Garcia ${ }^{3}$

RESUMO: Objetivo: identificar e discutir diagnósticos de enfermagem aplicáveis a pessoas com paraplegia em fase inicial de reabilitação domiciliar baseado na taxonomia da NANDA-I. Método: abordaram-se qualitativamente diagnósticos identificados no cuidado a três participantes. Estes foram organizados e agrupados em Domínios, explorando suas associações, variações, fatores relacionados e características definidoras. Resultados: domínios como Atividade/Repouso (Domínio 4); Enfrentamento/Tolerância ao estresse (Domínio 9) e Segurança/Proteção (Domínio 11) estão fortemente relacionados com traumatismos da medula espinhal, considerando-se que 0 Domínio 4 envolve diagnósticos para mobilidade, deslocamentos e desempenho de atividades para autocuidado; o Domínio 9 aborda respostas ao enfrentamento de situação estressora; e o Domínio 11, apresenta diagnósticos relacionados à presença de lesões ou com vulnerabilidade para elas. Conclusão: o processo de diagnóstico desenvolvido no domicílio facilitou a abordagem do contexto sociocultural, ambiental e emocional.

Descritores: Enfermagem; Diagnóstico de enfermagem; Traumatismos da medula espinhal; Paraplegia; Reabilitação; Assistência domiciliar.

ABSTRACT: Aim: to identify and to discuss nursing diagnoses applicable to people with paraplegia in initial phase of home rehabilitation based on Taxonomy of NANDA-I. Methods: it approached qualitatively diagnoses identified in care performed with three participants. They were organized and grouped within Domains and it was explored its associations, variations, related factors and defining characteristics. Results: domains like Activity/Rest (Domain 4); Coping/Stress Tolerance (Domain 9) and Safety/Protection (Domain 11) are deeply related with spinal cord injuries; this is considering that Domain 4 involves diagnoses about mobility, displacement and performance of activities for self-care; Domain 9 discusses responses to coping of stressful situation, and Domain 11 presents diagnosis related to presence of lesions or vulnerability for them. Conclusion: the diagnostic process developed at home facilitated the approach of socio-cultural, environmental and emotional context.

Descriptors: Nursing; Nursing diagnosis; Spinal cord injuries; Paraplegia; Rehabilitation; Home nursing.

RESUMEN: Objetivo: identificar y discutir diagnósticos de enfermería aplicables a personas con

\footnotetext{
${ }^{1}$ Enfermeira. Mestre em Enfermagem pela Universidade Federal do Ceará. Professora Assistente 1 da Universidade Federal de Sergipe. São Cristóvão, SE, Brasil. E-mail: karenineholanda@gmail.com

${ }^{2}$ Enfermeira. Pós-Doutora em Enfermagem pela Universidade Nova Lisboa/Portugal. Professor Associado 4 do Programa de Pós-Graduação em Enfermagem da Universidade Federal do Ceará. Fortaleza, CE, Brasil. E-mail: zmfca@fortalnet.com.br

${ }^{3}$ Enfermeiro. Especialista em Saúde do Trabalhador. Professor Auxiliar II da Universidade Federal de Mato Grosso. Cuiabá, MT, Brasil. E-mail: francisco.ufmt@gmail.com
} 


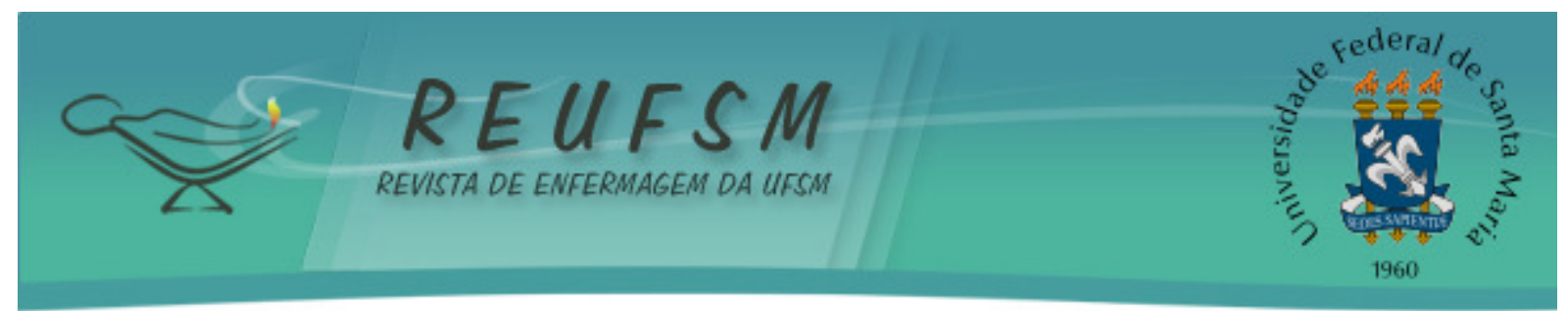

paraplejia en fase inicial de rehabilitación domiciliaria basado en la Taxonomía de la NANDA-I. Métodos: se utilizó cualitativamente diagnósticos identificados, que fueran agrupados en dominios, explorando sus asociaciones, variaciones, factores relacionados y características

definitorias. Resultados: dominios como Actividad/Descanso (Dominio 4); Enfrentamiento/Tolerancia al Estrés (Dominio 9) y Seguridad/Protección (Dominio 11) están profundamente relacionados con lesiones de médula espinal, teniendo en cuenta que el Dominio 4 incluye diagnósticos acerca de movilidad, desplazamiento y realización de actividades para autocuidado; Dominio 9 considera respuestas de enfrentamiento a situaciones estresantes, y Dominio 11, presenta diagnósticos relacionados con presencia de lesiones o vulnerabilidad para ellos. Conclusión: el proceso de diagnóstico desarrollado en el hogar facilitó el abordaje del contexto socio-cultural, ambiental y emocional.

Descriptores: Enfermería; Diagnóstico de enfermería; Traumatismos de la médula espinal; Paraplejía; Rehabilitación; Atención Domiciliaria de Salud.

\section{INTRODUÇÃO}

Devido à complexidade da lesão medular, o paciente demanda assistência específica de equipe interdisciplinar, tanto na fase aguda quanto na reabilitação. ${ }^{1} 0$ portador de traumatismo da medula espinhal (TME) deve receber os devidos cuidados não só em nível hospitalar, mas até a sua completa reintegração social. Faz-se necessário conhecimento acerca dos hábitos e estilo de vida no contexto social e domiciliar, para que haja direcionamento do cuidado integral. ${ }^{2}$ A fase inicial de reabilitação domiciliar, neste estudo, trata-se do período situado entre a alta hospitalar (fase aguda) e o ingresso em uma instituição de reabilitação.

Alguns países desenvolvem o programa de reabilitação no domicílio, assim, cliente e família participam ativamente para o alcance do máximo de independência funcional. ${ }^{3} \mathrm{O}$ enfermeiro é coordenador da prática de cuidar no processo de reabilitação. Mediante o Processo de Enfermagem, desenvolve um plano assistencial para operacionalizar o cuidado, manter um ótimo nível de saúde e prevenir complicações. Além disso, ajuda o paciente a identificar potenciais e desenvolver metas. ${ }^{4}$ Observa-se a importância da utilização de ferramentas próprias da enfermagem na assistência. Entretanto, as especificidades dessas pessoas exigirão direcionamento da assistência e adequação às etapas da reabilitação.

Um trabalho publicado em 1996, envolvendo sistematização da assistência de enfermagem (SAE) ao paciente com TME, já enfatizava dificuldade na redação de diagnósticos e metas de enfermagem, por pouca familiarização dos enfermeiros com as classificações de enfermagem, devido à carência na aplicabilidade pelos profissionais brasileiros. ${ }^{5}$ Pesquisas mostram que o uso sistemático da Taxonomia da NANDA-I facilita a comunicação entre enfermeiros, oferece linguagem padronizada, obtendo, assim, resultados desejáveis para o paciente, e contribui para a aquisição da autonomia no trabalho. ${ }^{6}$

Nesse contexto, esta pesquisa objetivou a identificação e discussão de diagnósticos de enfermagem (DEs), fundamentados na Taxonomia da NANDA-I, aplicáveis a pessoas com paraplegia na fase inicial de reabilitação domiciliar. A carência de estudos que versam sobre a temática do cuidado no TME envolvendo a SAE, torna este significativo para a Enfermagem, promovendo seu crescimento e autonomia enquanto profissão e ciência. Com isso, enfermeiros poderão promover um cuidado mais direcionado às necessidades reais das pessoas com paraplegia, de forma sistemática, proporcionando maior independência, autorrespeito e qualidade de vida. 


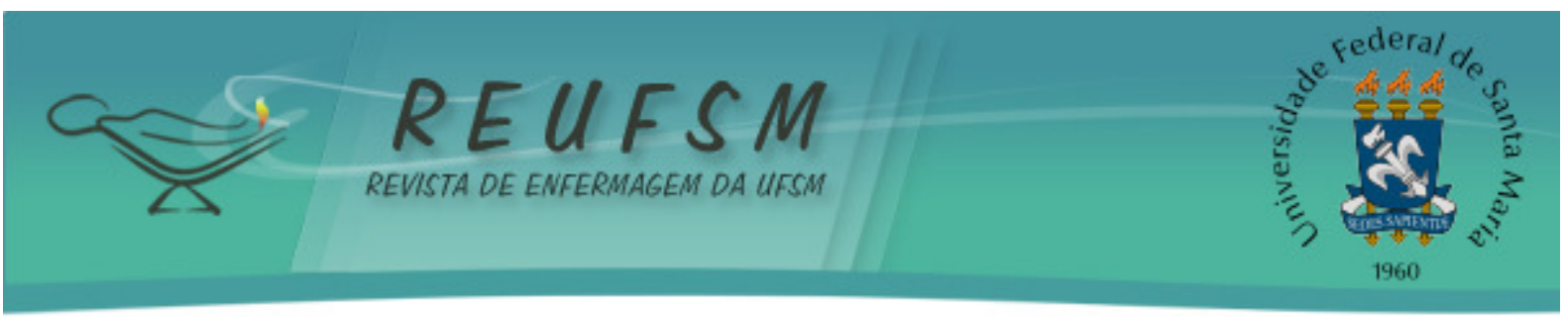

MÉTODO

Trata-se de uma pesquisa aplicada com caráter descritivo, que aborda qualitativamente os DEs levantados durante o cuidado efetuado em dez visitas domiciliárias por sujeito, entre abril e agosto de 2007. Participaram do estudo três pessoas, duas mulheres e um homem, adultos, com TME do tipo paraplegia e residentes em Fortaleza-CE-Brasil. Os critérios de inclusão foram: paraplegia; qualquer sexo, idade entre 18 e 45 anos; residir em Fortaleza-CE; e não submissão a programa de reabilitação de uma instituição.

0 Processo de Enfermagem orientou a coleta de dados por meio de entrevista, exame físico, observações e informações adquiridas de familiares, conforme proposto na primeira etapa do processo. Depois de identificadas as necessidades, problemas, riscos e bem-estar, respeitando as particularidades contextuais de cada um, foram propostos diagnósticos, utilizando a Taxonomia II da NANDA-I (2007-2008), que é estruturada em 13 Domínios, 47 Classes, contendo 187 Diagnósticos de Enfermagem. ${ }^{7}$

Para a análise dos dados, os DEs identificados foram organizados e agrupados por Domínios; sendo examinadas as associações e variações destes e de seus fatores relacionados e características definidoras. Posteriormente, foi feito um aprofundamento nas respostas destas pessoas ao TME no desempenho das atividades de vida, bem como os fatores responsáveis pelas alterações, buscando valores e interpretando à luz da literatura vigente.

Foram respeitados os preceitos éticos da Resolução 196/96 do Ministério da Saúde e o projeto foi aprovado pelo Comitê de Ética em Pesquisa da Universidade Federal do Ceará (Protocolo COMEPE N²42/07).

\section{RESULTADOS}

Os dados levantados foram discutidos valorizando-se detalhadamente as informações, entretanto, sem fazer referência ao sujeito fonte do dado. Foi considerado que, muitas vezes, diagnósticos diferentes estão relacionados, e intervenções estabelecidas para um destes pode sanar o outro, evitando-se planos extensos. Dos 13 Domínios observados na Taxonomia utilizada, estiveram presentes dez: Promoção da saúde (Domínio 1), Nutrição (Domínio 2), Eliminação/Troca (Domínio 3), Atividade/Repouso (Domínio 4), Percepção/Cognição (Domínio 5), Auto-Percepção (Domínio 6), Relacionamentos de papel (Domínio 7), Sexualidade (Domínio 8), Enfrentamento/Tolerância ao estresse (Domínio 9) e Segurança/Proteção (Domínio 11).

No primeiro Domínio, "Promoção da saúde", foi identificada "Manutenção ineficaz da saúde". As evidências encontradas foram: alimentação carente de nutrientes; água ingerida sem tratamento adequado; utilização de pia única para lavar roupas e louças; lençóis lavados e não passados a ferro e não submissão à consulta ginecológica há mais de um ano. Os fatores relacionados foram: falta de conhecimento e falta de recursos materiais.

No Domínio “Nutrição" esteve presente: "Risco de volume de líquidos deficiente" relacionado à ingestão deficiente de líquidos.

No Domínio “Eliminação/Troca” foi aplicado “Constipação", evidenciado por fezes endurecidas e períodos prolongados sem evacuações; devido à interrupção na transmissão nervosa de impulsos relacionados aos estímulos intestinais, à imobilidade, à ingesta insuficiente de fibras e líquidos e à falta de conhecimento acerca do programa de reeducação intestinal.

O Domínio 4, “Atividade/Repouso", mostrou-se fortemente afetado: “Atividade de recreação deficiente", "Capacidade de transferência prejudicada", "Déficit no autocuidado para alimentação, para higiene íntima e para vestir-se/arrumar-se", "Padrão de sono perturbado" e "Risco de Síndrome do desuso". A ausência de 


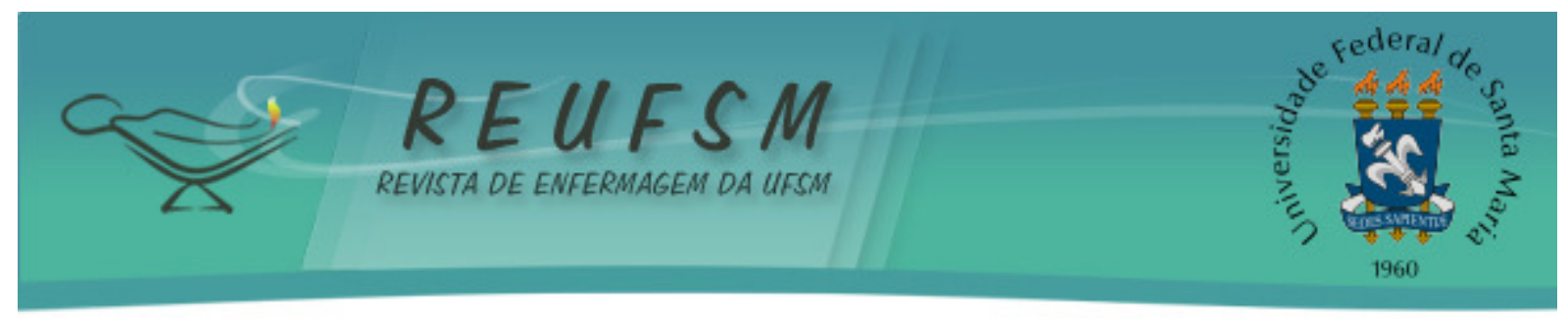

atividades recreativas teve como fatores relacionados: prejuízos neuromusculares, dificuldade de manipulação da cadeira de rodas e inacessibilidade arquitetônica pública.

A capacidade de transferência prejudicada pôde ser evidenciada pela dificuldade relatada e observada na passagem da cama para a cadeira de rodas ou vice-versa, e da cadeira de rodas para a cadeira higiênica ou vice-versa. Esta foi relacionada com o prejuízo neuromuscular; espasticidade; lesões ósseas, devido a traumas paralelos; e déficit de orientação de técnicas para transferência.

Quanto ao "Déficit de autocuidado", como se trata de paraplegia, na qual a parte alta do sistema digestivo está preservada, a negligência quanto à alimentação se deu pela dificuldade ou impossibilidade de preparar as próprias refeições como habitual. 0 déficit para ações de limpeza corporal e para vestir-se ou arrumar-se foi manifestado pela dependência de familiares, pois anteriormente eram realizadas com autonomia e privacidade, resultando em higiene deficiente e uso de vestimentas insuficientes ou inapropriadas. "Déficit no autocuidado para higiene íntima" foi caracterizado pela não realização do autocateterismo relacionada à carência de orientações sobre técnicas facilitadoras.

O sono fragmentado e/ou perturbado com pesadelos foi identificado e costuma estar presente até que a pessoa esteja reabilitada, tendo como fatores relacionados: não poder dormir na posição habitual, desconforto por permanência prolongada na mesma posição, desconforto no pescoço e esquentamento nas costas. Já o risco de síndrome do desuso surgiu pela carência de gerenciamento adequado por meio de um programa de atividades físicas, passivas e ativas.

No Domínio "Percepção/Cognição" foram aplicados: "Comunicação verbal prejudicada", "Conhecimento deficiente acerca de: possibilidade de febre como indicativo de infecção; utilização de manobras específicas para facilitar a eliminação vesical; alívio de pressão na cadeira de rodas; riscos de acidentes e complicações do TME e das reais consequências do TME que sofreu". Os DEs sobre conhecimento deficiente foram evidenciados pela verbalização de desconhecimento ou pela observação de inabilidade, e por comportamentos insuficientes na busca de saúde; tendo como fator relacionado falta de orientação. Quanto à comunicação verbal foram evidenciados: vocabulário reduzido, respostas curtas e retraimento; e foram relacionadas a déficit de conhecimento, baixo nível de escolaridade e timidez.

O sexto Domínio refere-se à "Auto-percepção" e teve como títulos de DEs: "Baixa autoestima situacional" e "Distúrbio de imagem corporal". As evidências de baixa autoestima foram expressões faciais e corporal de desânimo e verbalizações autodepreciativas; relacionadas a prejuízos neuromusculares que desencadeiam dependência para realização de atividades triviais, perda de autonomia, mudanças de papéis sociais e distúrbios de imagem corporal. O distúrbio de imagem corporal foi relacionado às alterações físicas e funcionais que desencadeiam questionamentos e confusões mentais acerca do próprio corpo. Foram encontradas as características definidoras: mudança real na estrutura e função, comportamento de evitar o próprio corpo, foco na função do passado e descontentamento com o atual estilo de vida.

0 Domínio 7, que abrange relacionamentos de papel, esteve presente por meio do DE com título "Maternidade prejudicada". O estudo mostrou a maternidade prejudicada pelas consequências físicas e psicológicas do TME em uma mãe, sendo evidenciada pela mudança de comportamento da filha com a mãe e demais familiares.

No Domínio 8 identificou-se "Padrões de sexualidade ineficazes" evidenciado pela não realização de atos sexuais e verbalização de descrença na possibilidade; resultando dos fatores: desconhecimento sobre as reais consequências do TME na função sexual; e falta de privacidade e diálogo com o companheiro a esse respeito.

No Domínio "Enfrentamento/Tolerância ao estresse" foram identificados: 


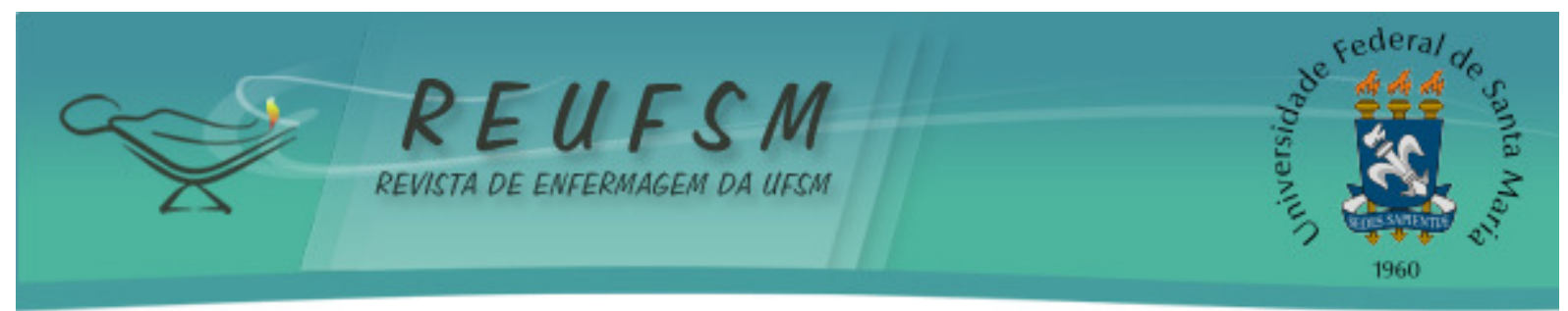

"Ansiedade", "Comportamento de saúde propenso à risco"e "Risco de disreflexia autonômica". A ansiedade foi identificada pela verbalização de desejos imediatistas relacionados à cura de úlcera por pressão, à admissão no hospital de reabilitação e ao retorno da capacidade de andar, relacionado a desconhecimento sobre as reais sequelas do TME, as mudanças que enfrentará no estilo vida ou mesmo seu prognóstico.

Para o julgamento de "Comportamento de saúde propenso à risco" evidenciou-se: pouca adaptação observada à nova situação e ausência de comportamento de busca de saúde ou prevenção de problemas; causados pelos fatores relacionados: desejo de não incomodar familiares, mudança de papéis e mudança de ambiente domiciliar.

Foram identificados como fatores de risco para disreflexia autonômica:estímulos dolorosos/irritativos abaixo do nível da lesão, distensão da bexiga, cateterização, distensão intestinal, menstruação, úlcera por pressão e espasmo.

No Domínio 11, "Segurança/Proteção" os DEs observados foram: "Integridade da pele prejudicada", "Risco de infecção", "Risco de queda" e "Risco de lesão". A integridade da pele prejudicada foi proveniente de traumas corriqueiros e de pressão prolongada na pele, desencadeando úlceras por pressão.

0 risco de lesão surgiu pela mobilidade alterada e o déficit sensório-motor, podendo causar arranhões, cortes e queimaduras. 0 risco de queda foi considerado devido às mobilizações e transferências. Fatores de risco para infecções foram: alteração nas funções vesical e intestinal; cateterismo vesical; rompimento da integridade da pele; dificuldades na tosse, em TME torácica; e conhecimento insuficiente acerca de prevenção de exposição a patógenos.

\section{DISCUSSÃO}

Mesmo passada a fase aguda, a pessoa demanda atenção especial para sua saúde como um todo. Em geral, há um intervalo de tempo entre a alta hospitalar recebida na fase aguda da lesão na medula espinhal e o ingresso em um programa de reabilitação. Pode-se dizer que a fase inicial de adaptação domiciliar é um período muito delicado pelo fato de ser o primeiro contato com as atividades de vida após o traumatismo. Portanto, se faz pertinente um programa de cuidados de equipe multiprofissional direcionado às peculiaridades da pessoa e seu contexto ambiental e sociocultural.

A reabilitação domiciliar inicial envolve percalços, pois exige mudanças familiares, ambientais, sociais e culturais; contudo, caracteriza-se por promover superação e emancipação. ${ }^{8}$

O corpo ainda se encontra debilitado, necessitando medidas de prevenção de infecção por meio de higiene, nutrição adequada e acompanhamentos por profissionais da saúde. Destaca-se que para preservação da saúde da mulher, a consulta ginecológica deve ser mantida. A manutenção ineficaz da saúde refere-se à "incapacidade de identificar, controlar e/ou buscar ajuda para manter a saúde". 7:249 Após o evento do TME as pessoas costumam voltar-se apenas para a imobilidade ou para problemas esfincterianos. Contudo, nessa fase de reabilitação domiciliar, o enfermeiro tem um especial favorecimento para abordar o paciente de forma holística por estar em contato com o contexto cotidiano e ambiental da pessoa e, assim, pode estimular a busca por melhores condições de bem-estar. ${ }^{9}$

A carência financeira como fator relacionado é uma situação real para muitos portadores de TME no Brasil, nos levando a refletir sobre a relevância de programas públicos de reabilitação, para o fornecimento do material e condições necessárias para este processo. Afinal, como manter nutrição ou higiene ideal, quando não se tem o mínimo indispensável para isto?

0 cuidado realizado no domicílio permite a percepção real do fator financeiro, evitando prescrições de enfermagem inadequadas às condições econômicas da pessoa.

Cabe ressaltar, que muitas vezes essas pessoas, antes do trauma, encontravam-se 


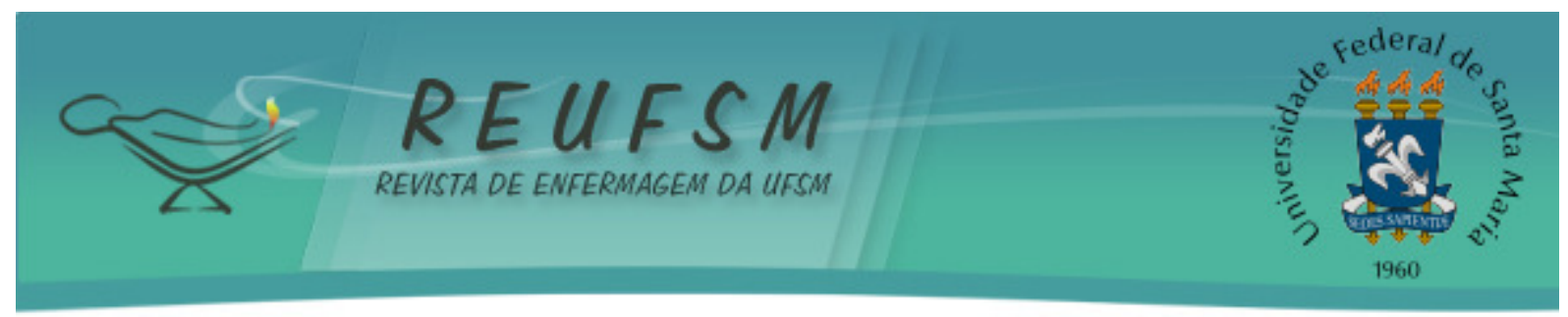

em fase ativa de trabalho, resultando, obviamente, em um impacto no aspecto econômico familiar. Um estudo com 156 casos de TME mostrou que 68,9\% tinham menos de quatro salários mínimos de renda familiar. ${ }^{10}$ Apresentou que $93,9 \%$ tinham ocupação antes do TME, e, após este, apenas 12,1\% mantiveram, 3,4\% mudaram de atividade, 17,4\% recebiam benefício, $15.4 \%$ estavam aposentados e $51,7 \%$ foram definidos como sem ocupação. ${ }^{10}$

Especialmente na situação inicial de reabilitação, será identificado déficit de conhecimento em diversos aspectos. Contudo, a falta de conhecimento originando problemas de enfermagem ratifica a necessidade de orientação. Educação em saúde feita no domicílio pode facilitar o direcionamento e contextualização, permitindo eficaz apreensão dos ensinamentos. A reorganização da pessoa e dos envolvidos na sua rede sócio-familiar depende de orientações claras e consistentes. ${ }^{11}$

Neste trabalho muitos DEs aplicados tiveram como fator relacionado falta de conhecimento, explicitando despreparo para alta hospitalar e ausência de participação em programa de reabilitação.

A identificação do diagnóstico de enfermagem e a assistência para carência de conhecimento é facilmente desenvolvida quando o processo de reabilitação está inserido no ambiente domiciliar do cliente.

Seja pela perda da sensibilidade ou mobilidade ou pelas disfunções autonômicas, a pessoa encontra-se mais vulnerável a traumas, infecções e úlceras. 0 conhecimento da febre como um sinal de infecção, de técnicas para alívio de pressão da cadeira de rodas no corpo e dos riscos de acidentes e quedas, proporciona prevenção de importantes complicações. Pesquisas revelam a alta incidência de úlceras por pressão em pessoas com TME, acrescentando que podem desencadear osteomielite, septicemia e mesmo óbito. ${ }^{12}$ Portanto, os envolvidos devem conhecer a fisiopatologia da lesão, suas consequências reais e possíveis complicações; e manobras que facilitam a eliminação vesical, evitando complicações.

O lar da pessoa com lesão medular pode ser um ambiente ideal para o ensino de técnicas e atitudes que previnam complicações e promovam independência, pois além de apresentar a situação realmente vivenciada, facilitando o uso de ferramentas próprias, a pessoa provavelmente se sentirá mais à vontade e receberá o apoio de familiares.

A úlcera por pressão, além do desconforto físico e risco de complicações que proporciona, costuma desencadear desordens emocionais e impedir ou dificultar a participação da pessoa em programas de reabilitação. ${ }^{12}$ No Brasil, comumente, instituições que promovem programas de reabilitação exigem ausência dessas lesões para iniciar o processo.

Além da presença de úlceras de pressão, não saber as reais sequelas do TME, as mudanças que enfrentará no estilo vida ou mesmo seu prognóstico, que costuma ser uma incógnita até mesmo para a equipe de saúde, comumente são fatores geradores de ansiedade. Portanto, a detecção do problema e assistência às úlceras por pressão ainda no domicílio é fundamental, sendo estas originadas antes ou após a alta hospitalar. Nesse sentido, serão avaliadas as ferramentas disponíveis como: presença e preparo do cuidador, produtos adequados para curativo, temperatura e higiene do ambiente e do leito, entre outros.

"Comportamento de Saúde Propenso à Risco" é uma "incapacidade de modificar estilo de vida/comportamentos de forma compatível com mudanças no estado de saúde". 7:56 A mudança de papéis e redução na autonomia exigem rápida adaptação ou mesmo reconstrução de papéis. Comumente, o acometido encontra-se na fase adulta, e exerce relevantes papéis familiares e sociais. A "Maternidade prejudicada" surge pela impossibilidade do cuidador primário em proporcionar crescimento e desenvolvimento adequado à criança.

Tais papéis exercidos e os membros familiares envolvidos são mais bem identificados e compreendidos com a realização da assistência na própria residência, facilitando o direcionamento do plano de cuidados.

A condição de paraplegia favorece o risco de Síndrome do desuso, que é definida 


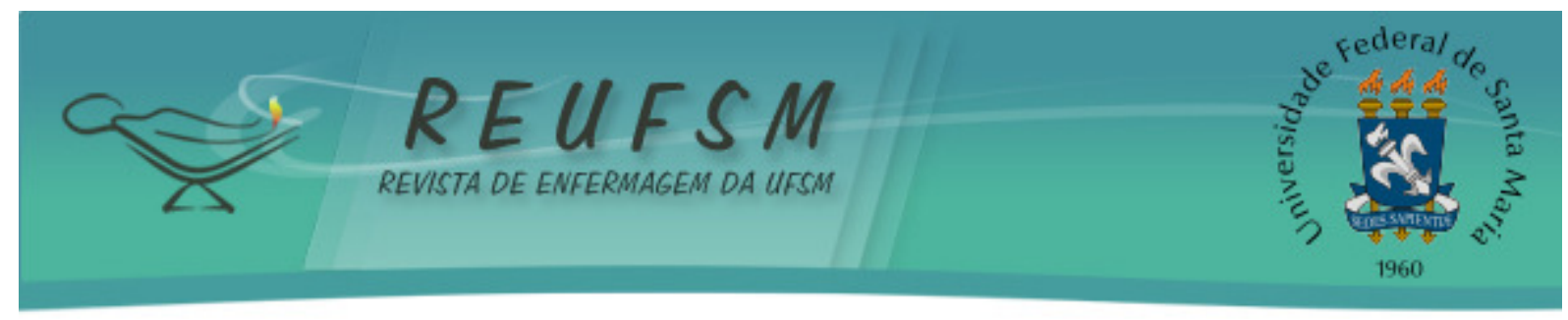

como "deterioração de sistemas do corpo como resultado de inatividade musculoesquelética"; ${ }^{7: 105}$ e de Disreflexia autonômica, que é uma resposta excessiva do sistema nervoso simpático pela ausência do controle do sistema parassimpático que pode ocorrer em pessoas com lesão medular acima do nível T6. ${ }^{3}$

Ingestão deficiente de líquidos aparece muitas vezes por falta de conhecimento acerca da importância da água para o bom funcionamento do organismo humano ou por falta de empenho na preservação da saúde. Entretanto, até adquirir um maior grau de independência, a pessoa com paraplegia depende de outros para the fornecer líquido. Com isso, percebe-se que baseado em diagnósticos de enfermagem adequados à reabilitação domiciliar, o plano de enfermagem demandará, a princípio, intensa participação da família.

Quanto à realização das habilidades de gerenciamento do intestino, espera-se destas pessoas a independência total após o processo de reabilitação. Porém, apesar de muitos conseguirem reconhecer quando vai evacuar (estímulo sensitivo, sudorese, piloereção), poucos se disponibilizam a realizar o treinamento diariamente. ${ }^{13}$

A inserção do enfermeiro no ambiente domiciliar permite a identificação de atitudes como a ingesta de líquidos e o gerenciamento intestinal e facilita a promoção da autorresponsabilização pela saúde.

Problemas voltados para atividade e repouso podem ser generalizados para pessoas que vivenciam a condição de paraplegia, devido à incapacidade inicial para mobilizar-se. Entretanto, espera-se que com um programa de reabilitação adequado, esses DEs sejam excluídos do plano de cuidados. A enfermeira avalia as dificuldades ambientais e a carência de equipamentos e direciona a busca por solução dos problemas.

A ausência de atividades recreativas, sejam elas já habituais ou novas, é uma tendência, pois culturalmente, os familiares e os próprios acometidos encaram essa situação como um "aprisionamento", reduzindo ou até eliminando o convívio social e o lazer. Entre os empecilhos destaca-se a pouca acessibilidade arquitetônica. Apesar da existência de políticas públicas no Brasil voltadas para a garantia da acessibilidade, esta ainda não é uma realidade.

Cuidar de si próprio contribui para a adaptação psicológica à nova condição e melhora a autoestima. A dependência de familiares gera constrangimentos, reduzindo a motivação para a reabilitação. Autocateterismo, realizado respeitando os princípios científicos, tem vantagens: reduz frequência de infecções, preserva a função vesical e estimula a reeducação, favorece a independência, conserva o sistema urinário e, principalmente, oferece a oportunidade de convívio social, pois possui intervalos determinados. ${ }^{13}$

Baixa autoestima surge quando a pessoa desenvolve visão negativa de seu valor, desencadeada por determinada situação presente, tornando-se amplamente aplicável nesta situação, já que prejuízos neuromusculares desencadeiam dependência para realização de atividades triviais, perda de autonomia, mudanças de papéis sociais e distúrbios de imagem corporal. É importante ratificar que mesmo ainda não sendo possível identificar a baixa autoestima como real, deve-se incluí-la no plano assistencial como diagnóstico de risco. Sentimentos de desamparo e falta de controle sobre si costumam ser descritos por essa população. Percepção da vida como externamente controlada promove menor autoestima. ${ }^{14}$

O distúrbio de imagem corporal pode não estar presente na fase inicial do processo de reabilitação, visto que a pessoa pode ainda estar na fase de negação da adaptação emocional. Contudo, as alterações físicas e funcionais desencadeiam questionamentos e confusões mentais acerca do próprio corpo. A inabilidade para caminhar e o estigma social com a deficiência, resultante do padrão social de beleza e produtividade, desencadeiam sentimentos de vergonha e inferioridade. ${ }^{15}$

A mulher com paraplegia, apesar da redução na sensibilidade da genitália e na lubrificação, poderá ter relações sexuais com a ajuda de soluções lubrificantes e de estímulos em 


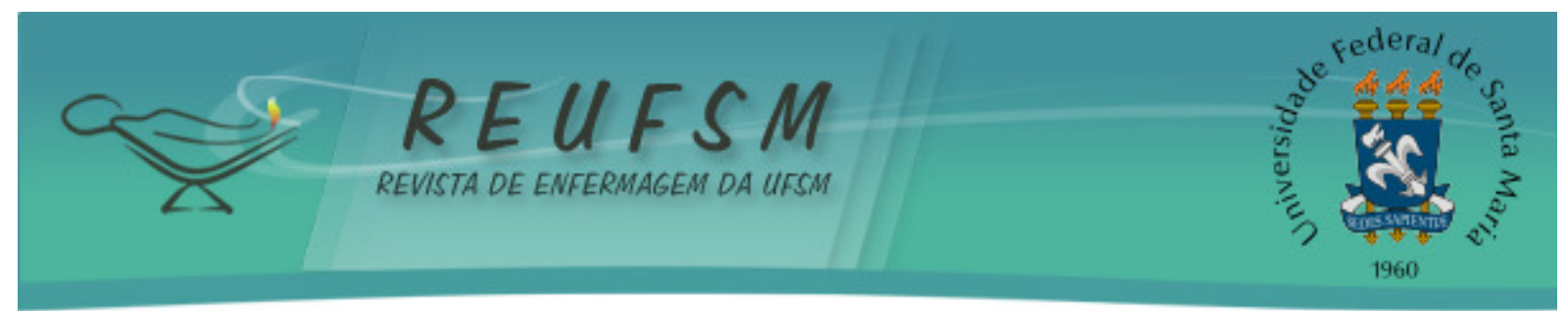

outras áreas corporais passíveis de prazer, e terá sua fertilidade preservada. 0 homem, dependendo do nível e grau da lesão, poderá apresentar algum tipo de ereção, proveniente de estímulo físico ou psicológico. O maior prejuízo estará na ejaculação, afetando sua fertilidade. ${ }^{16-17}$

Apesar deste estudo, tratar-se de uma assistência precoce, diagnósticos voltados para a sexualidade não devem ser excluídos, pois a lesão medular gera transtornos físicos e emocionais neste aspecto do ser humano que podem resultar em transtornos mentais secundários. Além disso, o domicílio é um ambiente estratégico para a detecção de problemas e intervenção voltada para o alcance da independência no aspecto sexualidade.

A comunicação é uma importante ferramenta de trabalho para a enfermagem. Comunicação verbal prejudicada pode afetar negativamente todo um programa de reabilitação, tornando-o ineficiente. Devem-se utilizar medidas para favorecer o conforto e a liberdade, tratar a pessoa com empatia, e adaptar a assistência ao seu nível cognitivo.

\section{CONCLUSÃO}

O estudo possibilitou identificação de problemas e necessidades na condição de paraplégico, bem como, a utilização de uma classificação de enfermagem para a realização do planejamento de cuidado específico.

A complexidade da interferência do TME no ser humano e suas funções gera uma vasta magnitude de necessidades e respostas, que demanda um amplo planejamento de enfermagem. Além da individualidade no contexto e formas de enfrentamento, o tempo decorrido após o acidente e a fase reabilitacional serão diferenciais na assistência de enfermagem.

0 processo diagnóstico desenvolvido no domicílio facilitou a abordagem das informações, permitindo compreender o paciente como um todo, inclusive seu contexto sociocultural, ambiental e emocional, proporcionando, ainda, o conhecimento de fatores motivacionais ou não para a busca da saúde e o direcionamento das intervenções.

Determinados problemas de saúde e ferramentas de superação são mais facilmente identificadas no ambiente domiciliar. 0 enfermeiro terá acesso, entre outros fatores, à estrutura da casa, que facilitará o cuidado especialmente à atividade de mobilização da pessoa; à estrutura familiar, um importante elemento de apoio; e às condições econômicas, que serão mais bem compreendidas.

A assistência no lar favorece a prevenção de complicações em um momento de grande risco como esse pós-alta hospitalar e a promoção de cuidados de reabilitação inicial e preparativo para o ingresso no programa de reabilitação efetivo, e isto em um ambiente que proporciona conforto, segurança e privacidade para a pessoa.

Assim, destaca-se a necessidade de acompanhamento na atenção primária, mediante visitas domiciliares, por equipe de saúde competente. Propõe-se com isso preencher a lacuna existente no Brasil entre a assistência na fase aguda e o ingresso em programa de reabilitação.

\section{REFERÊNCIAS}

1. França ISX, Coura AS, França EG, Basílio NNV, Souto RQ. Qualidade de vida de adultos com lesão medular: um estudo com WHOQOL-bref. Rev Esc Enferm USP [Internet]. 2011 [acesso em 2013 jan 9];45(6):1364-71. Disponível em: http://www.scielo.br/pdf/reeusp/v45n6/v45n6a13.pdf.

2. Cavalcante KMH. Cuidado de enfermagem à pessoa com paraplegia fundamentado no modelo Roper-Logan-Tierney [dissertação]. Fortaleza, CE: Universidade Federal do Ceará, Departamento de Enfermagem; 2007. Disponível

em: http://www.repositorio.ufc.br:8080/ri/bitstream/123456789/1784/1/2007_dis_kmhcavalcante.pdf. 


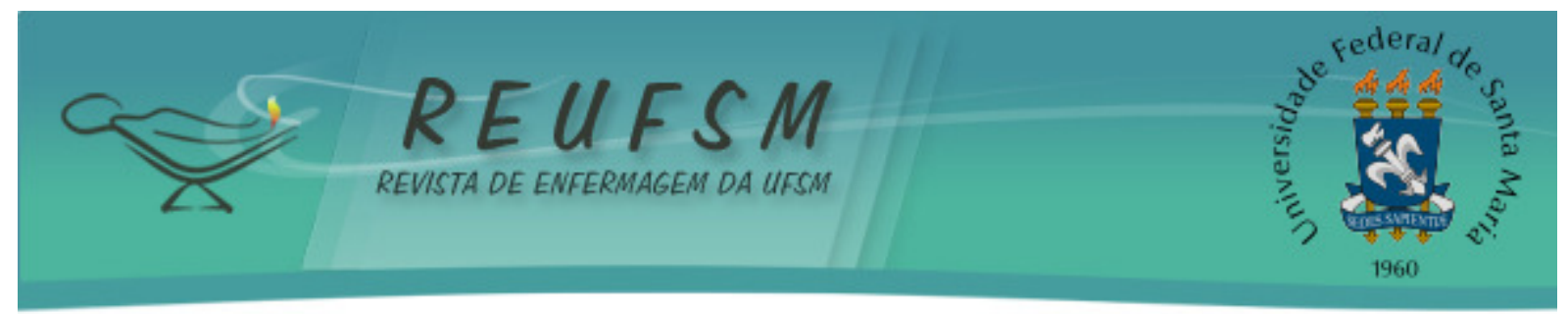

3. Hoeman SP. Rehabilitation nursing: process and application. $2^{\text {nd }}$ ed. St. Louis: Mosby; 1996.

4. Smeltzer SC, Bare BG, Hinkle JL, Cheever KH. Brunner \& Suddarth: tratado de enfermagem médico cirúrgico. 12 ${ }^{\mathrm{a}}$ ed. Rio de Janeiro (RJ): Guanabara Koogan; 2010.

5. Bocchi SCM, Meneguin S, Santi RC. Sistematização da assistência de enfermagem a paciente com luxação de coluna cervical: estudo de caso. Rev Latinoam Enferm [Internet]. 1996 [acesso em 2013 fev 4];4(2):113-29. Disponível em: http://www.scielo.br/pdf/rlae/v4n2/v4n2a09.pdf.

6. Cavalcante RB, Otoni A, Bernardes MFVG, Cunha SGS, Santos CS, Silva PC. Experiências de sistematização da assistência de enfermagem no Brasil: um estudo bibliográfico. Rev Enferm UFSM [Internet]. 2011 [acesso em 2013 fev 4];1(3):461-71. Disponível em: http://cascavel.ufsm.br/revistas/ojs-2.2.2/index.php/reufsm/article/view/2832/2396.

7. North American Nursing Diagnosis Association. Diagnósticos de enfermagem da NANDA: definições e classificação 2007-2008. Porto Alegre (RS): Artmed; 2008.

8. Marchi JA, Silva RH, Mai LD. O cuidado domiciliar a indivíduo com tetraplegia: um relato de experiência. Ciênc Cuid Saúde [Internet]. 2012 [acesso em 2013 abr 27];11(1):202-9. Disponível em: http://periódicos.uem.br/ojs/índex.php/CiencCuidSaude/article/view/18879.

9. Scramin AP, Machado WCA. Cuidar de pessoas com tetraplegia no ambiente domiciliário: intervenções de enfermagem na dependência de longo prazo. Esc Anna Nery Rev Enferm [Internet]. 2006 [acesso em 2013 abr 27];10(3):501-8. Disponível em: http://www.scielo.br/pdf/ean/v10n3/v10n3a20.pdf.

10. Noronha JB. Levantamento epidemiológico dos casos de lesão medular espinhal traumática atendidos em unidade de reabilitação de Goiânia-Goiás [dissertação]. Goiânia,GO: Universidade Católica de Goiás, Programa de Pós-Graduação em Ciências Ambientais e Saúde; 2008 [acesso em 2013 fev 7]. Disponível em: http: / / tede.biblioteca.ucg.br/tde_busca/arquivo.php?codArquivo=639.

11. Borges AMF, Brignol P, Schoeller SD, Bonetti A. Percepção das pessoas com lesão medular sobre a sua condição. Rev Gaúch Enferm [Internet]. 2012 [acesso em $2013 \mathrm{fev}$ 7];33(3):119-25. Disponível em: http://www.scielo.br/pdf/rgenf/v33n3/16.pdf.

12. Nogueira PC, Caliri MHL, Haas VJ. Profile of patients with spinal cord injuries and occurrence of pressure ulcer at a university hospital. Rev Latinoam Enferm [Internet]. 2006 [acesso em 2013 jan 7];14(3):372-7. Disponível em: http://www.scielo.br/pdf/rlae/v14n3/v14n3a10.pdf.

13. Venturini DA, Decesaro MN, Marcon SS. Conhecendo a história e as condições de vida de indivíduos com lesão medular. Rev Gaúch Enferm [Internet]. 2006 [acesso em 2013 jan 9];27(2):219-29. Disponível em: http://seer.ufrgs.br/RevistaGauchadeEnfermagem/article/view/4600/2520.

14. Brunozi AE, Silva AC, Gonçalves LF, Veronezi RJB. Qualidade de vida na lesão medular traumática. Rev Neurociênc [Internet]. 2011 [acesso em 2013 fev 8];19(1):139-44. Disponível em: http://www.revistaneurociencias.com.br/edicoes/2011/RN1901/revisao/444\%20revisao.pdf.

15. Costa VSP, Melo MRAC, Garanhani ML, Fujisawa DS. Social representations of the wheelchair for people with spinal cord injury. Rev Latinoam Enferm [Internet]. 2010 [acesso em 2013 fev 8];18(4):755-62. Disponível em: http://www.scielo.br/pdf/rlae/v18n4/14.pdf. 


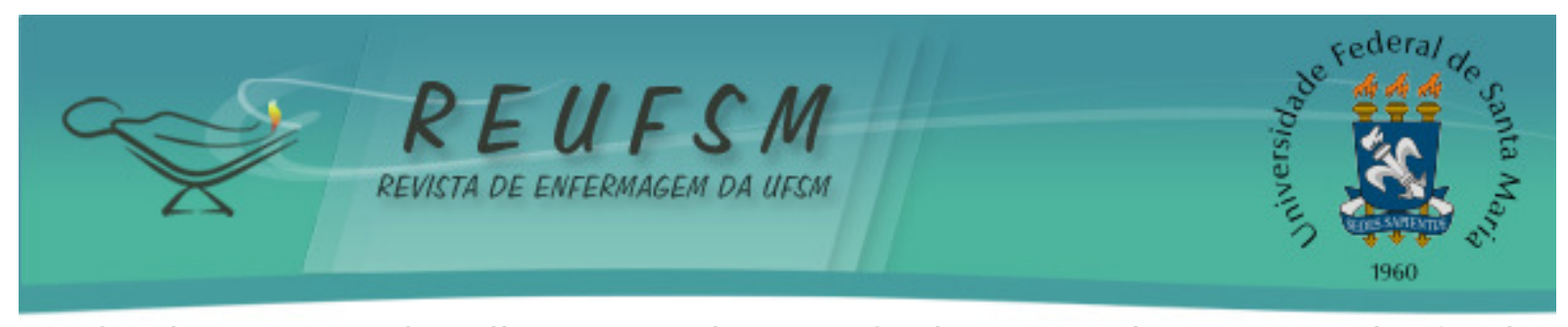

16. Cavalcante KMH, Carvalho ZMF, Barbosa IV, Studart RMB. Alterations in the fertility lived deeply by people with spinal cord injury: a qualitative research. Online Braz J Nurs [Internet]. 2007 [acesso em 2012 jan 7];6(3). Disponível em: http: / / www.objnursing.uff.br/index.php/nursing/article/view/j.1676-4285.2007.960/264.

17. Cavalcante KMH, Carvalho ZMF, Barbosa IV, Rolim GA. Vivência da sexualidade por pessoas com lesão medular. Rev RENE [Internet]. 2008 [acesso em 2013 fev 7];9(1):27-35. Disponível em: http://www.revistarene.ufc.br/revista/index.php/revista/article/viewFile/515/pdf.

Data de recebimento: $15 / 02 / 2013$

Data de aceite: 17/06/2013

Contato com autor responsável: Karenine Maria Holanda Cavalcante

E-mail: karenineholanda@gmail.com

Endereço postal: Rua Padre Álvares Pitangueira, $n^{\circ}$ 248. Centro, Lagarto, SE, Brasil.

CEP: $49100-000$ 